\title{
Risk assessments for broncho-pleural fistula and respiratory failure after lung cancer surgery by National Clinical Database Japan
}

\author{
Shunsuke Endo ${ }^{1,2} \cdot$ Norihiko Ikeda $^{1,3} \cdot$ Takashi Kondo $^{1,4} \cdot$ Jun Nakajima ${ }^{1,5} \cdot$ Haruhiko Kondo ${ }^{1,6} \cdot$ Yoshihisa Shimada $^{1,3}$. \\ Masami Sato ${ }^{1,7}$. Shinichi Toyooka ${ }^{1,8} \cdot$ Yoshinori Okada $^{1,9} \cdot$ Yukio Sato $^{1,10}$. Ichiro Yoshino ${ }^{1,11}$. Morihito Okada ${ }^{1,12}$. \\ Meinoshin Okumura ${ }^{1,13} \cdot$ Masayuki Chida ${ }^{1,14} \cdot$ Eriko Fukuchi $^{15} \cdot$ Hiroaki Miyata $^{15}$
}

Received: 12 August 2018 / Accepted: 5 October 2018 / Published online: 16 October 2018

(c) The Author(s) 2018

\begin{abstract}
Background Broncho-pleural fistula (BPF) and respiratory failure (RF) are life-threatening complications after lung cancer surgery and can result in long-term hospitalization and decreased quality of life. Risk assessments for BPF and RF in addition to mortality and major morbidities are indispensable in surgical decision-making and perioperative care.

Methods The characteristics and operative data of 80,095 patients who had undergone lung cancer surgery were derived from the 2014 and 2015 National Clinical Database (NCD) of Japan datasets. After excluding 1501 patients, risk models were developed from these data and validated by another dataset for 42,352 patients derived from the 2016 NCD dataset. Receiver operating characteristic curves were generated for postoperative BPF and RF development. The concordance-index was used to assess the discriminatory ability and validity of the model.

Results BPF and RF occurred in $259(0.3 \%)$ and 420 patients (0.5\%), respectively, in the model development dataset and in $129(0.3 \%)$ and 198 patients $(0.5 \%)$, respectively, in the model validation dataset. Characteristic variables including types of surgery and comorbidities were identified as risk factors for BPF and RF, respectively. The concordance indexes of assessments for BPF and RF were $0.847(p<0.001)$ and $0.848(p<0.001)$, respectively, for the development dataset and 0.850 $(p<0.001)$ and $0.844(p<0.001)$, respectively, for the validation dataset.

Conclusions These models are satisfactory for predicting BPF and RF after lung cancer surgery in Japan and could guide preoperative assessment and optimal measures for preventing BPF and RF.
\end{abstract}

Keywords Lung cancer surgery $\cdot$ Broncho-pleural fistula $\cdot$ Respiratory failure $\cdot$ Risk assessment $\cdot$ Nationwide survey

\section{Introduction}

Lung cancer is a leading cause of death worldwide. Surgery remains the mainstay for complete cure. Several risk models and scoring systems for improving stratification of mortality risk associated with lung cancer surgery have been developed and tested [1-3].

Broncho-pleural fistula (BPF) and respiratory failure (RF) are still major devastating postoperative complications after pulmonary resections for primary lung cancer even though

Electronic supplementary material The online version of this article (https://doi.org/10.1007/s11748-018-1022-y) contains supplementary material, which is available to authorized users.

Shunsuke Endo

tcvshun@jichi.ac.jp

Extended author information available on the last page of the article their incidences have decreased over the last two decades. Both are strongly associated with high mortality and negative impacts on activities of daily living following longterm hospitalization. Several risk factors for BPF and RF as male, performance status, some comorbidities, and extensive resection, etc., have been suggested in patients undergoing pulmonary resection [4-6]. No existing risk ratio integrated by these risk factors responds to such medical conditions as development of surgical devices and less invasive surgeries in these days. Availability of a risk calculator at the time of surgery in response to a real-time medical condition would enable identification of surgical indications, improve surgical outcomes, maintain patient performance after surgery, and reduce medical costs, especially when the surgical candidate has multiple risk factors. 


\section{Patients and methods}

\section{Data collection}

In 2011, the NCD of Japan adopted an annual web-based nationwide data collection system. The Japan Surgical Society ethics committee reviewed and approved the ethical characteristics of the entire National Clinical Database initiative and fully disclosed the review process, on its website. The use of registry data for retrospective observational studies was approved by the committee, and the requirement of individual written or verbal informed consent was waived because of the retrospective design. In 2014, data on 1.6 million surgical procedures from more than 4000 hospitals were collected [7]. An NCD specifically for general thoracic surgery was launched in 2014. The data registration system and information recorded are described in detail in our previous report [8]. In total, 80,095 lung cancer operations were registered in the 2014 and 2015 datasets. More than $95 \%$ of all pulmonary resections for lung cancer registered with the Regional Bureau of Health and Welfare in Japan are accounted for in the NCD [9].

These databases have been audited regularly by a webauditing system since 2015. The audit encompasses other variables related to patient demographics and outcomes, these having been evaluated using hospitalization summaries randomly selected by the NCD office since 2017 . The high quality of the database has enabled development of risk models for BPF and RF after lung cancer surgery [1].

\section{Patient cohort}

The cohort for the current analysis was derived from three annual datasets $(2014,2015$, and 2016) that included information on persons who underwent surgical resection for primary lung cancer (at 797 surgical units in 2014, 814 in 2015, and 814 in 2016). Surgical approaches were categorized as thoracotomy or minimally invasive, including complete video-assisted thoracoscopic surgery and mini-thoracotomy with a wound length of $8 \mathrm{~cm}$ or less. Variables related to surgical approach were excluded in the risk analysis because decisions regarding surgical approach varied among surgical units. The surgical procedures included wedge resection, segmentectomy, lobectomy, bilobectomy, and pneumonectomy. Bronchoplasty (sleeve- or wedge-plasty) and extensive resections including chest wall, pulmonary artery, diaphragm, and so on were also entered into the analysis. Nodal dissection was categorized as hilar, lobe-specific mediastinal, or systematic dissection.

Patients were excluded if they had undergone procedures with no curative intent, extrapleural pneumonectomy, complete pneumonectomy, emergency surgery, combined procedures for both lungs, or been transported by ambulance. Of the 80,095 patients in 2014 and 2015 and the 43,017 in 2016, 1,501 and 665 patients were excluded from the analysis, respectively. Thus, the development dataset for risk models included 78,594 patients entered in 2014 and 2015 , and the validation dataset 42,352 patients entered in 2016.

\section{Outcome measures}

The primary outcome measures were BPF and RF occurring during the index hospitalization or within 30 days after surgery regardless of hospitalization status. BPF was defined as communication between the pleural space and bronchial tree with/without empyema. Causes of RF after lung cancer surgery range from atelectasis and pneumonia to acute lung injury. The NCD system [8] defines RF as when a patient requires tracheal intubation or tracheostomy or mechanical ventilation for $48 \mathrm{~h}$ or longer after lung cancer surgery regardless of cause, such as acute exacerbation of interstitial pneumonia, pneumonia, atelectasis, pulmonary emboli, and so on.

\section{Statistical analysis}

We divided the cohort into a training set (cases from 2014 to 2015) and a validation set (cases from 2016). We constructed a multivariable logistic regression model that predicted the incidences of BPF and RF from patient demographic variables, comorbidities, and tumor conditions. Missing values for age and spirometry were replaced with the most frequent category. The estimates from the fitted models were then used to predict the risk for each patient in the testing cohort, based on the following equation: Predicted BPF $(\mathrm{RF})=e\left(b_{0}\right.$ $\left.+\Sigma b_{i} X_{i}\right) / 1+e\left(b_{0}+\Sigma b_{i} X_{i}\right)$, where $b_{i}$ is the coefficient of the variable $X_{i}$ in the logistic regression equation provided in the tables. $X_{i}=1$ if a categorical risk factor is present and 0 if it is absent. We categorized age into six levels and included it as a continuous variable. $X_{i}=0$ for a patient age less than $60, X_{i}=1$ for age $60-64, X_{i}=2$ for age $65-69, X_{i}=3$ for age 70-74, $X_{i}=4$ for age $75-79$, and $X_{i}=5$ for an age greater than 79. We assessed the discrimination of the model by examining the area under the receiver operating characteristic curve (C-statistic) and its calibration by a calibration plot and the Hosmer-Lemeshow test. All analyses were performed with the IBM SPSS Statistics software package (version 24; IBM, Armonk, NY, USA). 


\section{Results}

\section{Risk profile of study cohort}

From 1 January 2014 through 31 December 2015, 78,594 patients underwent lung cancer surgery (risk model set) and from 1 January 2016 through 31 December 2016, 42,352 (validation set). The baseline characteristics of these patient groups were similar (Supplemental Table S1).

\section{Outcomes}

BPF and RF occurred in $259(0.3 \%)$ and 420 patients $(0.5 \%)$, respectively, in the model development dataset and in 129 $(0.3 \%)$ and 198 patients $(0.5 \%)$, respectively, in the model validation dataset. The 30 day and in-hospital mortality of the patients who developed BPF and RF were $18.9 \%$ and $35.7 \%$, respectively, in the model development dataset and $19.4 \%$ and $38.4 \%$, respectively, in the model validation dataset. Reoperation for the patients with BPF was performed in 135 patients $(52.1 \%)$ in the model development dataset and 65 patients $(50.4 \%)$ in the model validation dataset. Length of hospitalization was $35.3 \pm 29.1$ days in the patients with $\mathrm{BPF}$, which is significantly longer than that of patients without $\mathrm{BPF}(11.3 \pm 10.1$ days; $p<0.001)$. It was $40.7 \pm 28.5$ days in the patients with RF, which is significantly longer than that in patients without $\mathrm{RF}(11.3 \pm 10.0$ days; $p<0.001)$ (Supplemental Tables S2 and S3).

\section{Model results}

Multivariate risk models were developed and the final logistic model, with odd ratios (ORs) and 95\% confidence intervals (CIs), is presented in Tables 1 and 2, which show the associations of patient baseline characteristics with the outcome measures of BPF and RF occurrence. 17 variables were associated with BPF occurrence and 27 with RF occurrence.

To evaluate model performance, the concordance-index (a measure of model discrimination), which is the area under the receiver operating characteristic curve, was used. The C-indices were 0.847 (95\% CI $0.824-0.869 ; p<0.001)$ for BPF and 0.848 (95\% CI $0.831-0.865 ; p<0.001)$ for RF. The $\mathrm{C}$-indices in the validation datasets for BPF were 0.850 (95\% CI, 0.819-0.881; $p<0.001$ ) and 0.844 (95\% CI 0.816-0.871; $p<0.001$ ) for RF. Figure 1 shows the calibration of the models and the extent to which the rates for the predicted events matched those of the observed events among the patient risk subgroups.
Table 1 Predictors of bronchopleural fistula

\begin{tabular}{llll}
\hline & $\beta$ coefficient & $p$ value & $\begin{array}{l}\text { Mortality model } \\
\text { OR (95\% CI) }\end{array}$ \\
\hline Sex male & 1.014 & $<0.001$ & $2.756(1.760-4.315)$ \\
BMI under 18.5 & 0.928 & $<0.001$ & $2.529(1.834-3.488)$ \\
PS2 or over & 0.670 & 0.004 & $1.954(1.231-3.102)$ \\
Interstitial pneumonia & 0.485 & 0.020 & $1.624(1.079-2.446)$ \\
Central nerve system disorder & 0.693 & $<0.001$ & $2.000(1.375-2.910)$ \\
Autoimmune disease & 1.026 & 0.001 & $2.789(1.558-4.996)$ \\
Cigarette smoking habit & 0.659 & 0.007 & $1.934(1.199-3.118)$ \\
Induction radiotherapy or chemoradiotherapy & 0.806 & 0.003 & $2.238(1.309-3.826)$ \\
Clinical N1or higher & 0.498 & 0.002 & $1.645(1.202-2.252)$ \\
Clinical stage IB or higher & & 0.012 & $1.505(1.096-2.068)$ \\
Surgical procedure & 0.409 & & \\
Right pneumonectomy & & $<0.001$ & $11.070(6.518-21.001)$ \\
Right lower lobectomy & 2.460 & $<0.001$ & $4.102(3.137-5.365)$ \\
Left pneumonectomy & 1.412 & 0.026 & $2.451(1.114-5.395)$ \\
Bronchoplasty with segmentectomy or lobectomy & 1.679 & $<0.001$ & $5.358(3.438-8.358)$ \\
Hilar nodal dissection or more & 1.317 & 0.001 & $3.733(1.732-8.047)$ \\
Combined resection & & & \\
Chest wall resection & 0.926 & 0.005 & $2.524(1.315-4.844)$ \\
Wedge resection or segmentectomy of lung & 0.722 & 0.011 & $2.058(1.178-3.596)$ \\
Intercept $(\beta 0)$ & -9.476 & & \\
\hline
\end{tabular}

$B P F$ broncho-pleural fistula, $C I$ confidence interval, FEV1 forced expiratory volume in $1 \mathrm{~s}, P S$ performance status, $V C$ vital capacity

${ }^{\mathrm{a}}$ Clinical staging was clarified by 7 th lung cancer classification 
Table 2 Predictors of respiratory failure

\begin{tabular}{|c|c|c|c|}
\hline & $\beta$ coefficient & $p$ value & $\begin{array}{l}\text { Mortality model } \\
\text { OR }(95 \% \mathrm{CI})\end{array}$ \\
\hline Sex male & 0.476 & 0.004 & $1.595(1.163-2.188)$ \\
\hline Age category ${ }^{a}$ & 0.219 & $<0.001$ & $1.245(1.156-1.341)$ \\
\hline BMI under 18.5 & 0.738 & $<0.001$ & $2.091(1.613-2.710)$ \\
\hline BMI over 30 & 0.682 & 0.008 & $1.978(1.198-3.265)$ \\
\hline Performance status 1 & 0.629 & $<0.001$ & $1.875(1.499-2.345)$ \\
\hline Performance status 2 or higher & 0.724 & $<0.001$ & $2.063(1.452-2.930)$ \\
\hline$\%$ VC $10 \%$ decrease $(100-50 \%)$ & 0.308 & $<0.001$ & $1.361(1.273-1.455)$ \\
\hline FEV $1 \% 10 \%$ decrease $(100-50 \%)$ & 0.550 & $<0.001$ & $1.734(1.343-2.239)$ \\
\hline Liver cirrhosis (Child-Pugh B/C) & 1.323 & $<0.001$ & $3.756(1.807-7.808)$ \\
\hline Interstitial pneumonia & 1.107 & $<0.001$ & $3.024(2.312-3.956)$ \\
\hline Coronary artery disease & 0.422 & 0.006 & $1.524(1.127-2.062)$ \\
\hline Central nerve system disorder & 0.575 & $<0.001$ & $1.776(1.342-2.352)$ \\
\hline Arrhythmia & 0.610 & 0.001 & $1.840(1.301-2.603)$ \\
\hline Cigarette smoking 30 pack-years or more & 0.462 & 0.001 & $1.587(1.214-2.074)$ \\
\hline Tumor size $>3 \mathrm{~cm}$ & 0.343 & 0.005 & $1.410(1.108-1.793)$ \\
\hline Clinical T1b or higher ${ }^{\mathrm{b}}$ & 0.299 & 0.025 & $1.349(1.038-1.753)$ \\
\hline Clinical T3 or higher ${ }^{\mathrm{b}}$ & 0.350 & 0.033 & $1.419(1.028-1.980)$ \\
\hline Clinical stage III or higher ${ }^{\mathrm{b}}$ & 0.386 & 0.011 & $1.471(1.092-1.958)$ \\
\hline Histology squamous cell carcinoma & 0.369 & 0.001 & $1.447(1.166-1.795)$ \\
\hline Superior sulcus tumor & 0.516 & 0.022 & $1.675(1.079-2.601)$ \\
\hline Multiple lung cancers & 0.984 & $<0.001$ & $2.675(1.722-4.157)$ \\
\hline \multicolumn{4}{|l|}{ Surgical procedure } \\
\hline Right pneumonectomy & 1.538 & $<0.001$ & $4.655(2.507-8.642)$ \\
\hline Right upper lobectomy & 0.350 & 0.002 & $1.418(1.135-1.772)$ \\
\hline Bilobectomy & 1.425 & $<0.001$ & $4.159(2.223-7.282)$ \\
\hline Bronchoplasty with segmentectomy or lobectomy & 0.651 & 0.009 & $1.917(1.174-3.131)$ \\
\hline Hilar nodal dissection or more & 0.813 & $<0.001$ & $2.254(1.591-3.193)$ \\
\hline \multicolumn{4}{|l|}{ Combined resection } \\
\hline Chest wall resection (other than first rib) & 0.857 & 0.001 & $2.356(1.447-3.835)$ \\
\hline Chest wall resection (first rib) & 1.257 & 0.004 & $3.515(1.492-8.282)$ \\
\hline Intercept $(\beta 0)$ & -9.741 & & \\
\hline
\end{tabular}

$C I$ confidence interval, $F E V 1$ forced expiratory volume in $1 \mathrm{~s}, R F$ respiratory failure; VC: vital capacity

${ }^{a}$ The variable of age was categorized into six groups, namely less than 60,60 to 64,65 to 69,70 to 74,75 to 79 , and more than 80 years old. Therefore, this odds ratio indicates an alteration of relative risk per one unit increase in age category

${ }^{\mathrm{b}}$ Clinical staging was clarified by 7 th lung cancer classification

\section{Discussion}

Several risk models and scoring systems derived by analyzing a big database have been developed and tested to improve stratification of mortality risk in lung cancer surgery [1-3]; however, a few studies have dealt only with BPF and/or RF risks [4-6]. The incidence of BPF is reportedly $0.6-4.4 \%$ and the mortality rate very high $(18-50 \%)$, although its incidence has decreased over the last two decades [5]. The incidence of pulmonary complications ranging from atelectasis to respiratory failure requiring mechanical ventilation is reportedly $10-20 \%$ after lung cancer surgery [10]. BPF and $\mathrm{RF}$ can both occur in the same patient. Development of BPF and/or RF is associated with high mortality and negative impacts on the patient's daily life despite lengthy hospitalization and large medical costs.

Various risk factors ranging from patient's characteristics to operative procedures have been reported to be associated with the development of BPF or RF. Few studies, however, have used multivariate analysis to assess integrated risk calculation of various factors on large clinical databases. Most previous studies had small patient cohorts, few BPF or RF events, and biased patient selection and surgical procedures, among other limitations.

Risk assessments for mortality and/or major morbidities after pulmonary resection for primary lung cancer using 

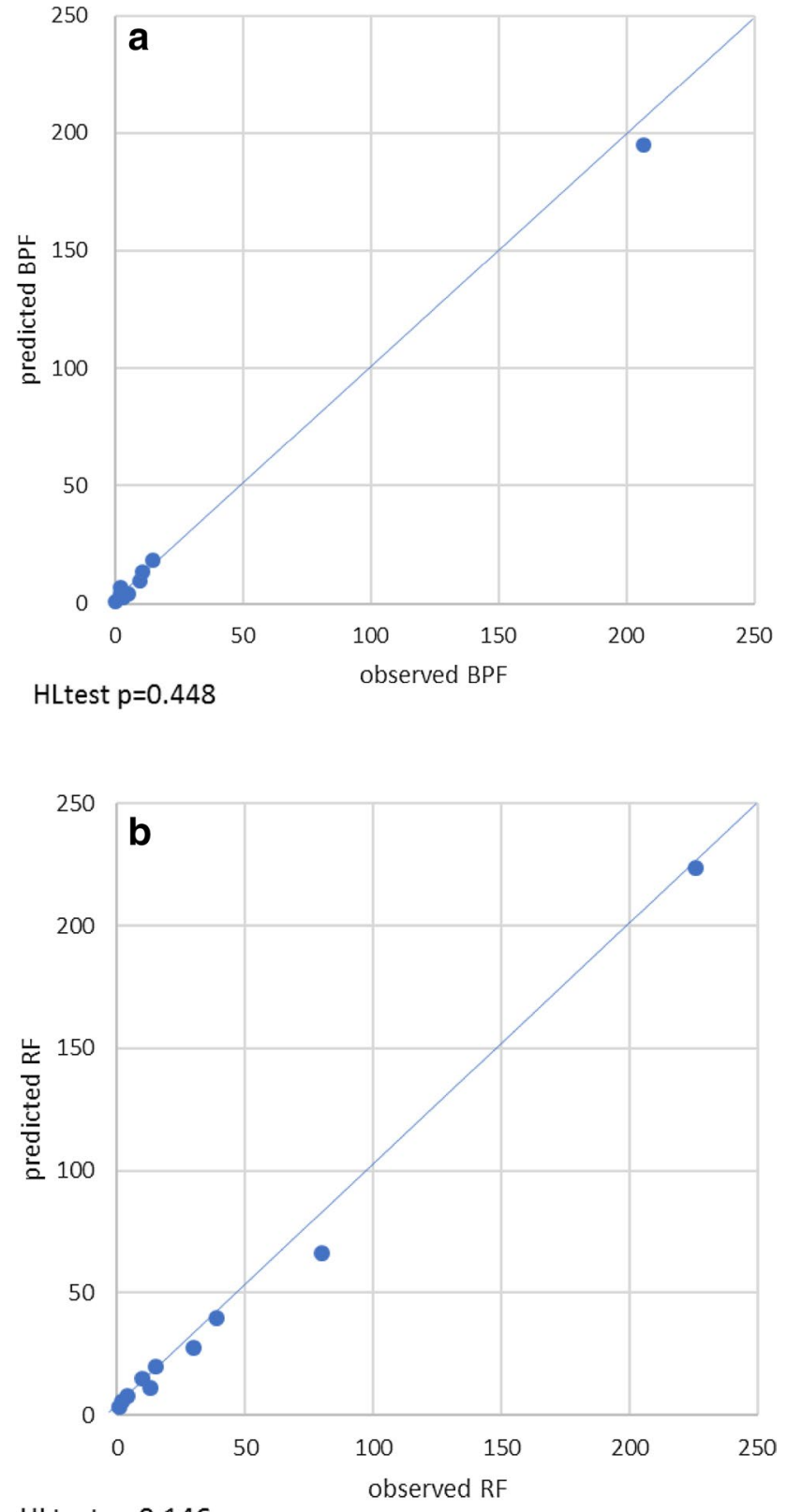

HLtest $p=0.146$

Fig. 1 Calibration of the broncho-pleural fistula (BPF) (a) and respiratory failure (RF) (b) models

approximately 80,000 surgery data from NCD were developed in the previous paper [1]. In this study, we additionally developed a model for estimating risk of BPF and RF using approximately 120,000 data derived from NCD for surgical decision-making and perioperative care. The NCD for lung cancer surgery provides a large number of lung cancer patients annually (approximately 40,000) who have undergone pulmonary resection, allowing identification of some variables as independent predictors of BPF and RF. Furthermore, these factors were validated using datasets obtained in 2016.
When surgeons input preoperative data and the planned surgery type, a BPF and RF risk models can improve preoperative assessment, including determination of surgical indications and the suitability of limited resection. It can also recommend individualized perioperative management as perioperative treatment or rehabilitation.

\section{Risk factors for BPF}

Male sex has consistently been identified as a risk factor in other models of lung cancer surgery risk [11]. Being underweight may be linked to sarcopenia, immune-nutritional deficiencies, and wound healing problems [12]. The American Society of Anesthesiologists physical status, which is estimated by number of comorbidities and their severity, may be a more useful indicator than performance status of development of pulmonary complications in older patients being considered for thoracoscopic lobectomy [13]. The NCD system requires entry of all comorbidities, thus enabling our identification of interstitial pneumonia, stroke, and autoimmune disease as comorbidities significantly associated with risk of BPF. Interstitial pneumonia is a known major risk factor for operative mortality after lung cancer surgery [1]. Acute exacerbation of interstitial pneumonia causing respiratory failure and requiring mechanical ventilation may be linked to development of BPF [14]. Autoimmune disease is possibly linked to immunosuppressive treatments such as steroids and/or potential delays in wound healing. Whether such immunosuppressive treatments should be continued should be carefully discussed with physicians [15].

Several studies have concluded that diabetes mellitus (DM) is a risk factor for BPF [16]; however, we did not confirm this in our study. DM status is categorized in the NCD system as follows: untreated, dietary treatment only, non-insulin treatment, and insulin-dependent. There are few patients with untreated DM in our series because we excluded patients requiring emergency surgery. Most patients' DM is now successfully controlled before surgery, even if they have severe DM. The presence of uncontrolled DM may be a risk factor for BPF. Neoadjuvant therapy is considered a major risk factor [17]. There has recently been an increased focus on radiation intervention in relation to BPF in studies of neoadjuvant therapy prior to surgery.

Pneumonectomy, especially right sided, is a known highrisk surgical procedure for BPF. Furthermore, right lower lobectomy has also been identified as a risk factor for BPF [18]. After both of these procedures the bronchial stump may be bare to the pleural cavity with minimal covering by mediastinal tissue or residual lung [5, 19]. Nodal dissection may strip the bronchial circulation, resulting in bronchial ischemia. Chest wall resection may adversely affect 
postoperative ventilation and bronchial circulation by stripping intercostal arteries.

\section{Prevention of BPF}

Bronchial stump coverage is the most frequently performed preventive procedure against BPF in high-risk patients; however, no randomized control studies have been conducted to clarify its efficacy as a preventive procedure against BPF. Some studies have reported that bronchial stump coverage is ineffective. In a prospective study of pneumonectomy in patients with DM, intercostal muscle flap coverage in highrisk patients was reported to confer significant benefit: no BPF occurred in the reinforcement arm [20]. Another study has reported a slightly higher incidence of BPF in high-risk patients who underwent bronchial stump coverage than in low-risk patients who did not [21]. Despite the lack of conclusive evidence, most thoracic surgeons believe that bronchial stump coverage can prevent from BPF. The extent of nodal dissection should also be considered.

Reoperation was saved in half of the patients with BPF. The result suggested that postoperative care of patients at high-risk of BPF must include close monitoring and urgent intervention to avoid surgical intervention for BPF [22].

\section{Risk factors for RF}

RF occurs due to pulmonary complications ranging from atelectasis and pneumonia to acute lung injury. Not only sarcopenia but obesity has been identified as a risk factor for RF. The spirometric variables of percent vital capacity $(\% \mathrm{VC})$ and forced expiratory volume 1.0\% (FEV1.0\%) are major risk factors for RF. Low $\% \mathrm{VC}$ and $\mathrm{FEV} 1.0 \%$ are risk indicators in patients with restrictive lung disease and chronic obstructive pulmonary disease (COPD), respectively. Recent studies have suggested the diffusion capacity of the lung for carbon monoxide (DLCO) is a more reliable predictor of RF in patients with restrictive lung disease [23]. The NCD system did not require data entry for DLCO until NCD 2016, because the DLCO test was infrequently performed in Japanese surgical units. Considering interstitial pneumonia is one of the risk factors for RF, DLCO will likely become a significant risk factor for RF in the near future, because it has been possible to enter DLCO since NCD 2017.

Organ failure such as hepatic dysfunction, ischemic heart disease, and central nerve disorder is also a risk factor for RF. Hepatic cirrhosis is a key prognostic factor in patients with acute respiratory distress syndrome [24].

Multiple lung cancers, which have increasingly been diagnosed since development of high-resolution chest computed tomography in this decade, are a risk factor for RF. Volume of resected lung may contribute to the RF risk likely in the patients undergoing right pneumonectomy or bilobectomy.

Few studies have investigated risk calculation of RF after pulmonary resection [6]. Risk of pulmonary complications is reportedly associated with administration of induction chemotherapy and predicted postoperative DLCO. Despite its simplicity, that model was found to be poor by external validation [25]. The model we developed from the 2014 and 2015 NCD datasets is surprisingly satisfactory according to the model validation dataset of NCD 2016 because we analyzed a dataset of approximately 80,000 lung cancer surgeries and the spectrum of RF was limited based on definition as a complication of atelectasis and pneumonia requiring tracheal intubation or tracheostomy or mechanical ventilation for $48 \mathrm{~h}$ or longer after lung cancer surgery regardless of complications such as acute exacerbation of interstitial pneumonia, pneumonia, atelectasis, pulmonary emboli, and so on [8].

\section{Prevention of RF}

Prediction of postoperative complications and long-term disability caused by pulmonary resection requires preoperative physiologic rehabilitation or treatment, to reduce a risk of respiratory failure for lung cancer patients with underlying pulmonary diseases such as idiopathic pulmonary fibrosis (IPF) [26] or chronic obstructive disease [27]. Planned surgical invasiveness as indicated by surgical approach, volume of resected lung, extent of nodal dissection, and extensive resection can have major physiological impacts on postoperative respiratory function in these patients. Less invasive approach as video-assisted thoracoscopic surgery or robotic surgery should be considered when risk calculation suggests high-risk [28, 29]. Palliative sublobar resection should be considered if applicable. Nodal dissection increases tissue damage by reducing lymphatic drainage and denervating the vagal nerve, thus increasing the pulmonary complication rate after lung cancer surgery [30]. Nodal sampling should be indicated by preoperative PET assessment or waived by nodal assessment through transbronchial lymph node biopsy.

Basic perioperative care including oxygen therapy, fluid management, use of bronchodilator and anti-IPF agent, and rehabilitation, etc., should be careful [31].

\section{Study limitations}

Our attempt to develop an ideal model for predicting risk of BPF and RF after lung cancer surgery has the following limitations.

1. The risk model was developed using a large amount of data from the NCD specifically concerning general tho- 
racic surgery in Japan. The NCD includes mostly a single race (Japanese), and contains few patients with high BMI, peripheral vascular disease, or untreated DM. The characteristics of enrolled patients may, therefore, differ from those of previous studies. Variations in risk models that are related to regional differences in data collection are of concern. In the future, we plan to develop a compatible risk calculator for other countries by streamlining the version for both models.

2. BPF may have been misdiagnosed and entered as prolonged pulmonary air leakage when the BPF was small. $\mathrm{BPF}$ is subject to entry error and under-reporting and this would have affected the model.

3. $\mathrm{BPF}$ and RF can develop over 30 days after surgery [32]. Late complications over 30 days after surgery are not entered into the NCD data entry system. Therefore, some patients with late onset of these complications may have been under-reported.

4. The NCD does not include fluid intake during surgery, which may be linked to RF, or operative procedures such as closure technique (manual or stapled), or bronchial covering, which may be associated with occurrence of BPF. These issues should be considered in future studies.

\section{Updated risk model}

Our analysis provided a risk model for BPF or RF after lung cancer surgeries based on the NCD 2014 and 2015 dataset. Validation analysis using the NCD 2016 dataset confirmed the feasibility of the risk model. However, patient characteristics, lung cancer oncology, treatment strategies, and development of surgical devices will continue to change as population age and medical science advances. Risk models for BPF and RF will, therefore, need to be reviewed, particularly in rapidly changing societies. Our annual data collection system of NCD will respond to these changes and enable updating of risk models for BPF and RF after lung cancer surgery [8].

In summary, models estimating risk of BPF and RF after lung cancer surgery based on the 2014 and 2015 NCD datasets precisely predicted occurrence of BPF and $\mathrm{RF}$ after lung cancer surgeries according to the $2016 \mathrm{NCD}$ dataset. The model can facilitate perioperative assessment and planning of operative procedures to prevent BPF and RF.

Acknowledgements This work was supported in part by a Health Labour Sciences Research Grant (No. 201313055C).

\section{Compliance with ethical standards}

Conflict of interest The authors have no conflicts of interest to report.

Open Access This article is distributed under the terms of the Creative Commons Attribution 4.0 International License (http://creativeco mmons.org/licenses/by/4.0/), which permits unrestricted use, distribution, and reproduction in any medium, provided you give appropriate credit to the original author(s) and the source, provide a link to the Creative Commons license, and indicate if changes were made.

\section{References}

1. Endo S, Ikeda N, Kondo T, Nakajima J, Kondo H, Yokoi K, et al. Model of lung cancer surgery risk derived from a Japanese nationwide web-based database of 78594 patients during 2014-2015. Eur J Cardiothorac Surg. 2017;52:1182-9.

2. Fernandez FG, Kosinski AS, Burfeind W, Park B, DeCamp MM, Seder C, et al. The Society of Thoracic Surgeons lung cancer resection risk model: higher quality data and superior outcomes. Ann Thorac Surg. 2016;102:370-7.

3. Brunelli A, Salati M, Rocco G, Varela G, Van Raemdonck D, Decaluwe H, et al. ESTS Database Committee. European risk models for morbidity (EuroLung1) and mortality (EuroLung2) to predict outcome following anatomic lung resections: an analysis from the European Society of Thoracic Surgeons database. Eur J Cardiothorac Surg. 2016;51:490-7.

4. Pforr A, Pagès PB, Baste JM, Thomas P, Falcoz PE, Lepimpec Barthes F, et al. Epithor Project French Society of Thoracic and Cardiovascular Surgery. A predictive score for bronchopleural fistula established using the French Database Epithor. Ann Thorac Surg. 2016;101:287-93.

5. Okuda M, Go T, Yokomise H. Risk factor of bronchopleural fistula after general thoracic surgery: review article. Gen Thorac Cardiovasc Surg. 2017;65:679-85.

6. Amar D, Munoz D, Shi W, Zhang H, Thaler HT. A clinical prediction rule for pulmonary complications after thoracic surgery for primary lung cancer. Anesth Analg. 2010;110:1343-8.

7. Miyata H, Gotoh M, Hashimoto H, Motomura N, Murakami A, Tomotaki A, et al. Challenges and prospects of a clinical database linked to the board certification system. Surg Today. 2014;44:1991-9.

8. Endo S, Ikeda N, Kondo T, Nakajima J, Kondo H, Yokoi K, et al. Development of an annually updated Japanese national clinical database for chest surgery in 2014. Gen Thorac Cardiovasc Surg. 2016;64:569-76.

9. Gotoh M, Miyata H, Hashimoto H, Wakabayashi G, Konno H, Miyakawa S, et al. National Clinical Database feedback implementation for quality improvement of cancer treatment in Japan: from good to great through transparency. Surg Today. 2016;46:38-47.

10. Allen MS, Darling GE, Pechet TT, Mitchell JD, Herndon JE 2nd, Landreneau RJ, et al ACOSOG Z0030 Study Group. Morbidity and mortality of major pulmonary resections in patients with early-stage lung cancer: initial results of the randomized, prospective ACOSOG Z0030 trial. Ann Thorac Surg. 2006;81:1013-9. (discussion 1019-20).

11. Thomas PA, Berbis J, Baste JM, Le Pimpec-Barthes F, Tronc F, Falcoz PE, et al. EPITHOR group. Pneumonectomy for lung cancer: contemporary national early morbidity and mortality outcomes. J Thorac Cardiovasc Surg. 2015;149:73.82. 
12. Thomas PA, Berbis J, Falcoz PE, Le Pimpec-Barthes F, Bernard A, Jougon J, et al. EPITHOR group. National perioperative outcomes of pulmonary lobectomy for cancer: the influence of nutritional status. Eur J Cardiothorac Surg. 2014;45:652-9. (discussion 659).

13. Zhang R, Kyriss T, Dippon J, Hansen M, Boedeker E, Friedel G. American Society of Anesthesiologists physical status facilitates risk stratification of elderly patients undergoing thoracoscopic lobectomy. Eur J Cardiothorac Surg. 2017. https://doi. org/10.1093/ejcts/ezx436. (Epub ahead of print).

14. Kobayashi S, Karube Y, Nishihira M, Inoue T, Araki O, Maeda S, et al. Postoperative pyothorax a risk factor for acute exacerbation of idiopathic interstitial pneumonia following lung cancer resection. Gen Thorac Cardiovasc Surg. 2016;64:476-80.

15. Busti AJ, Hooper JS, Amaya CJ, Kazi S. Effects of perioperative antiinflammatory and immunomodulating therapy on surgical wound healing. Pharmacotherapy. 2005;25:1566-91.

16. Li SJ, Fan J, Zhou J, Ren YT, Shen C, Che GW. Diabetes mellitus and risk of bronchopleural fistula after pulmonary resections: a meta-analysis. Ann Thorac Surg. 2016;102:328-39.

17. Takahashi S, Go T, Kasai Y, Yokomise H, Shibata T. Relationship between dose-volume parameters and pulmonary complications after neoadjuvant chemoradiotherapy followed by surgery for lung cancer. Strahlenther Onkol. 2016;192:658-67.

18. Shekar K, Foot C, Fraser J, Ziegenfuss M, Hopkins P, Windsor M. Bronchopleural fistula: an update for intensivists. J Crit Care. 2010;25:47.55.

19. Satoh Y, Okumura S, Nakagawa K, Horiike A, Ohyanagi F, Nishio $\mathrm{M}$, et al. Postoperative ischemic change in bronchial stumps after primary lung cancer resection. Eur J Cardiothorac Surg. 2006;30:172-6.

20. Sfyridis PG, Kapetanakis EI, Baltayiannis NE, Bolanos NV, Anagnostopoulos DS, Markogiannakis A, et al. Bronchial stump buttressing with an intercostal muscle flap in diabetic patients. Ann Thorac Surg. 2007;84:967-71.

21. Di Maio M, Perrone F, Deschamps C, Rocco G. A meta-analysis of the impact of bronchial stump coverage on the risk of bronchopleural fistula after pneumonectomy. Eur J Cardiothorac Surg. 2015;48:196-200.

22. Cardillo G, Carbone L, Carleo F, Galluccio G, Di Martino M, Giunti R, et al. The rationale for treatment of postresectional bronchopleural fistula: analysis of 52 patients. Ann Thorac Surg. 2015;100:251-7.

23. Brunelli A, Dinesh P, Woodcock-Shaw J, Littlechild D, Pompili C. Ninety-day mortality after video-assisted thoracoscopic lobectomy: incidence and risk factors. Ann Thorac Surg. 2017;104:1020-6.

24. Gacouin A, Locufier M, Uhel F, Letheulle J, Bouju P, Fillatre $\mathrm{P}$, et al. Liver cirrhosis is independently associated with 90-day mortality in ARDS patients. Shock. 2016;45:16-21.

25. Yepes-Temiño MJ, Monedero P, Pérez-Valdivieso JR. Grupo Español de Anestesia Toracica. Risk prediction model for respiratory complications after lung resection: an observational multicentre study. Eur J Anaesthesiol. 2016;33:326-33.

26. Iwata T, Yoshida S, Fujiwara T, Wada H, Nakajima T, Suzuki H, et al. Effect of perioperative pirfenidone treatment in lung cancer patients with idiopathic pulmonary fibrosis. Ann Thorac Surg. 2016;102:1905-10.

27. Takegahara K, Usuda J, Inoue T, Ibi T, Sato A. Preoperative management using inhalation therapy for pulmonary complications in lung cancer patients with chronic obstructive pulmonary disease. Gen Thorac Cardiovasc Surg. 2017;65:388-91.

28. Scott WJ, Allen MS, Darling G, Meyers B, Decker PA, Putnam $\mathrm{JB}$, et al. Video-assisted thoracic surgery versus open lobectomy for lung cancer: a secondary analysis of data from the american college of surgeons oncology group z0030 randomized clinical trial. J Thorac Cardiovasc Surg. 2010;139:976-81.

29. Kneuertz PJ, D'Souza DM, Moffatt-Bruce SD, Merritt RE. Robotic lobectomy has the greatest benefit in patients with marginal pulmonary function. J Cardiothorac Surg. 2018;13:56.

30. Okada M, Sakamoto T, Yuki T, Mimura T, Miyoshi K, Tsubota N. Selective mediastinal lymphadenectomy for clinico-surgical stage I non-small cell lung cancer. Ann Thorac Surg. 2006;81:1028-32.

31. Tsubochi H, Shibano T, Endo S. Recommendations for perioperative management of lung cancer patients with comorbidities. Gen Thorac Cardiovasc Surg. 2018;66:71-80.

32. Jichen QV, Chen G, Jiang G, Ding J, Gao W, Chen C. Risk factor comparison and clinical analysis of early and late bronchopleural fistula after non-small cell lung cancer surgery. Ann Thorac Surg. 2009;88:1589-93.

\section{Affiliations}

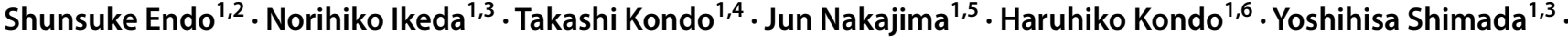 Masami Sato $^{1,7}$. Shinichi Toyooka ${ }^{1,8} \cdot$ Yoshinori Okada $^{1,9} \cdot$ Yukio Sato $^{1,10}$. Ichiro Yoshino ${ }^{1,11}$. Morihito Okada ${ }^{1,12}$. Meinoshin Okumura ${ }^{1,13} \cdot$ Masayuki Chida ${ }^{1,14} \cdot$ Eriko Fukuchi $^{15} \cdot$ Hiroaki Miyata $^{15}$}

1 Japanese Association for Chest Surgery, 3F Chiyoda Seimei Kyoto Oike Building 200 Takamiya-cho, Takakura-Oike-dori, Nakagyo-ku, 604-0835 Kyoto, Japan

2 Department of Thoracic Surgery, Jichi Medical University, Tochigi, Japan

3 Department of Thoracic Surgery, Tokyo Medical University Hospital, Tokyo, Japan

4 Department of Thoracic Surgery, Tohoku Medical and Pharmaceutical University Hospital, Miyagi, Japan

5 Department of Thoracic Surgery, University of Tokyo Graduate School of Medicine, Tokyo, Japan
6 Department of General Thoracic Surgery, Kyorin University Hospital, Tokyo, Japan

7 Department of General Thoracic Surgery, Graduate School of Medical and Dental Sciences, Kagoshima University, Kagoshima, Japan

8 Department of Thoracic Surgery, Graduate School of Medicine, Dentistry and Pharmaceutical Sciences, Okayama University, Okayama, Japan

9 Department of Thoracic Surgery, Institute of Development, Aging and Cancer, Tohoku University, Miyagi, Japan

10 Faculty of Medicine, Department of Thoracic Surgery, University of Tsukuba, Ibaraki, Japan 
11 Department of General Thoracic Surgery, Graduate School of Medicine, Chiba University, Chiba, Japan

12 Department of Surgical Oncology, Hiroshima University, Hiroshima, Japan

13 Department of General Thoracic Surgery, Osaka University Graduate School of Medicine, Osaka, Japan
14 Department of General Thoracic Surgery, Dokkyo Medical University, Tochigi, Japan

15 Department of Healthcare Quality Assessment, Graduate School of Medicine, University of Tokyo, 7-3-1 Hongo, Bunkyo-ku, 113-8655 Tokyo, Japan 